\title{
EXTENDABILITY OF CERTAIN MAPS
}

Dedicated to Professor Itiro Tamura on his 60-th birthday

\author{
By SeIYa Sasao
}

\section{$\S 1$. Introduction.}

In this note we will discuss the following problem:

Let $p: X \rightarrow S^{n}$ be a fibre bundle over the $n$-sphere having the fibre $A$ and the structure group $G$. Then what are obstructions for extending a given map

$$
f: A=p^{-1}\left(s_{0}\right) \rightarrow Y \text { over } X \text { ? }
$$

Usually an extension of a map is done step by step, i. e. skeltonwisely, in the homotopy theory. However this method is not successful except homotopically simple cases. In our case the situation is also the same, for example, a $C W$-decomposition of $X$ is not so clear in general. Then we try to attack the problem by the another way, and our purpose is to describe obstructions without using the space $X$ but spaces $A, Y$ and the characteristic class of the bundle.

We denote by $Y^{X}$ the space of all continuous maps: $X \rightarrow Y$ endowed with the Compact-Open topology. Roughly speaking our obstruction is in the homotopy group $\pi_{n-1}\left(Y^{A}, f\right)$. However it is not so easy to describe this group, then some restrictions are needed, for example, $Y$ is a topological group or a space which a group $H$ acts on, $\cdots$ etc.

Now let $p_{i}: X_{i} \rightarrow S^{n}(i=1,2)$ be principal $G_{i}$-bundles with its characteristic classes $\xi_{i}$ respectively. Then, as an application, we have

ThEorem. A map $f:\left(G_{1}, e_{0}\right) \rightarrow\left(G_{2}, e_{0}\right)$ is extendable to a map: $X_{1} \rightarrow X_{2}$ if and only if $f_{*}\left(\xi_{1}\right)=m \xi_{2}$ for some integer $m$ and

$$
o(f)_{*}\left(\xi_{1} \wedge 1_{G_{1}}\right) \in \partial\left[S^{n} \wedge G_{1}, S^{n}\right]_{0} \subset\left[S^{n-1} \wedge G_{1}, G_{2}\right]_{o}
$$

where $\wedge$ is the join operation and $o(f), \partial$ are explained in the context.

Andmoreovere, for example, we have

Received October 6, 1986 
COROLlary. Let $p_{i}: X_{i} \rightarrow S^{n}$ two principal $G_{i}$-bundles with its characteristic class $\xi_{\imath}(i=1,2)$ respectively. Assume that $\operatorname{dim} G_{2}$ is less than $n-1$ and $G_{1}, G_{2}$ are both path-connected. Then $X_{1}$ has the same homotopy type as $X_{2}$ if and only if there exists a homotopy equivalence $h: G_{1} \rightarrow G_{2}$ such that $h_{*}\left(\xi_{1}\right)= \pm \xi_{2}$ and $o(h)_{*}\left(\xi_{1} \wedge 1_{G_{1}}\right) \in \xi_{2^{*}}\left[S^{n-1} \wedge G_{1}, S^{n-1}\right]_{o}$.

Finally I thank prof. A. Dold and his coleague at Universität Heidelberg for their kindness in preparation of this note.

\section{$\S 2$. Obstructions.}

Let $p: X \rightarrow S^{n}$ be the fibre bundle as stated in $\S 1$. First we note the following lemma which is originally deviced in [1].

LEMMA 1. $X$ is considered as a space which is obtained from identification as follows.

$$
X=A \cup D^{n} \times A, \quad(x, a) \equiv \xi(x) a \quad \text { for } x \in S^{n-1}=\dot{D}^{n} .
$$

Proof. We may regard the disk $D^{n}$ as a space

$$
S^{n-1} \times I \cup D^{n}, \quad(x, 1) \equiv x \quad \text { for } x \in \dot{D}^{n}=S^{n-1} .
$$

Hence $X$ is decomposed as follows :

$$
\begin{gathered}
X=A \cup\left(\left(S^{n-1} \times I\right) \times A\right) \cup D^{n} \times A, \quad((x, 0), a) \equiv \xi(x) a \text { and } \\
\quad((x, 1), a) \equiv(x, a) \quad \text { for } x \in S^{n-1}=\dot{D}^{n} .
\end{gathered}
$$

Define a map $\phi: A \cup\left(S^{n-1} \times I\right) \times A \rightarrow D^{n} \times A$ by

$$
\begin{aligned}
& \psi(a)=(o, a) \quad(o=\text { the origin }) \\
& \phi((x, t), a)=(t x, \xi(x) a) .
\end{aligned}
$$

Clearly this is well defined and easily chequed to be homeomorphic. Thus $X$ may be considered the space

$$
D^{n} \times A \cup D^{n} \times A, \quad(x, a) \equiv(x, \xi(x) a) \quad \text { for } x \in S^{n-1}=\dot{D}^{n}
$$

i.e. This complets the proof.

Remark. We assume throughout this note that $A$ and all topological igroups are compact.

Now consider the commutative diagram of mappings : 


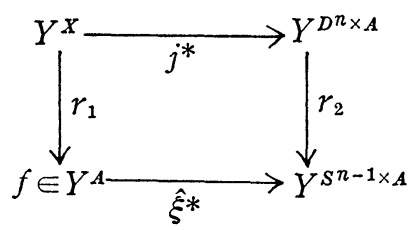

where $r_{1}, r_{2}$ are both fibrings obtained from the restriction of maps and $j: D^{n} \times A$ $\rightarrow X$ is the natural map and $\hat{\xi}: S^{n-1} \times A \rightarrow A$ is defined by $\hat{\xi}(x, a)=\xi(x) a$. Then from Lemma 1 we have

LEMMA 2. The diagram above is a pull-back one.

Then our idea is very simple, namely we only put

$$
Y^{A}=Z \text {. }
$$

The diagram is turned out the diagram:

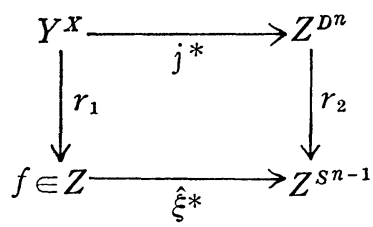

which shows that

$f$ is extendable over $X$

$\Longleftrightarrow$ there exists $\bar{f} \in Y^{X}$ such that $r_{1}(\dot{f})=f$

$\Longleftrightarrow \hat{\xi}^{*}(f) \in r_{2}$-image

$\Longleftrightarrow \hat{\xi}^{*}(f)$ is extendable over $D^{n}$ as a map

$\Longleftrightarrow \hat{\xi}^{*}(f):\left(S^{n-1}, s_{0}\right) \rightarrow(Z, f)$ is null as a base-point free map

$\Longleftrightarrow \hat{\xi}^{*}(f)=0$ in $\pi_{n-1}(Z, f)$

Thus we know that our obstruction is just $\hat{\xi}^{*}(f)$. Let $e_{0}$ be the unit of a group and $\phi$ be the action of $G$ on $A(G \times A \rightarrow A)$. We denote by $A_{0}^{A}, Y_{0}^{X}, \cdots$ etc. the subspace of $A^{A}, Y^{X}, \cdots$ etc. which consists of base-point preserving maps. Now the diagram gives rise to a new diagram:

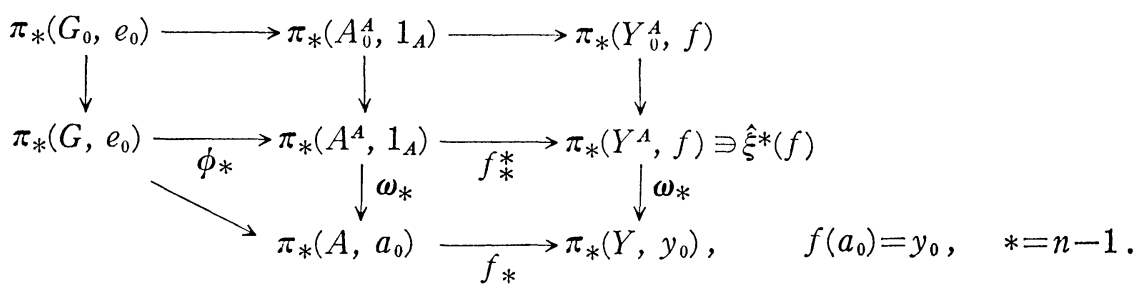


where $G_{0}$ is the isotropy subgroup of $G$ at $a_{0}$ and $\omega$ denotes the usual fibring.

Remark. The homomorphism $\pi_{*}\left(G, e_{0}\right) \rightarrow \pi_{*}\left(A, a_{0}\right)$ is induced from the map $G \rightarrow A\left(g \rightarrow g a_{o}\right)$ and the image of $\xi$ is just $\partial(\iota)$ for the boundary operator

$$
\partial: \pi_{n}\left(S^{n}\right) \longrightarrow \pi_{n-1}(A)
$$

associated with the fibre bundle.

For example, we know that $f_{*}(\partial \iota)=0$ if $\hat{\xi}^{*}(f)=0$, which means that $f$ can be extendable over $A \cup\left(D^{n} \times a_{0}\right)$ i.e. $f_{*}\left(\partial_{\iota}\right)$ is the first obstruction in the step by step method. Howevere, in general, we can't proceed any more because it is difficult to describe the group $\pi_{n-1}\left(Y^{A}, f\right)$. Thus we need some assumptions for computing $\pi_{n-1}\left(Y^{A}, f\right)$. We consider two cases.

\section{$\S 3$. The case of $Y$ being a topoogical group $H$.}

Let $\bar{y}$ be the constant map from any space to $Y$ whose value is $y$.

LEMMA 3. $\left(H^{A}, f\right)$ is homeomorphic to $\left(H_{o}^{A}, \bar{e}_{0}\right) \times\left(H, e_{0}\right), f\left(a_{o}\right)=e_{0}$.

Proof. First a homeomorphism $\alpha:\left(H^{A}, f\right) \rightarrow\left(H^{A}, \bar{e}_{0}\right)$ is defined by

$$
\alpha(s)(a)=s(a) f(a)^{-1} .
$$

Secondly define maps $\beta_{1}, \beta_{2}, \gamma_{1}$ and $\gamma_{2}$ as follows:

$$
\begin{array}{ll}
\beta_{1}:\left(H^{A}, \bar{e}_{0}\right) \longrightarrow\left(H_{0}^{A}, \bar{e}_{0}\right) & \beta_{1}(s)(a)=s(a) s\left(a_{0}\right)^{-1} \\
\beta_{2}:\left(H^{A}, \bar{e}_{0}\right) \longrightarrow\left(H, e_{0}\right) & \beta_{2}(s)=s\left(a_{0}\right) \\
\gamma_{1}:\left(H_{0}^{A}, \bar{e}_{0}\right) \longrightarrow\left(H^{A}, \bar{e}_{0}\right) & \gamma_{1}(s)=s \\
\gamma_{2}:\left(H, e_{0}\right) \longrightarrow\left(H^{A}, \bar{e}_{0}\right) & \gamma_{2}(a)=h .
\end{array}
$$

Then the map $\beta_{1} \times \beta_{2}:\left(H^{A}, \bar{e}_{0}\right) \rightarrow\left(H_{o}^{A}, \bar{e}_{o}\right) \times\left(H, e_{o}\right)$ is a homeomorphism. These show the proof.

Now, using Lemma 3, we have a decomposition of $\pi_{n-1}\left(H^{A}, f\right)$ as follows

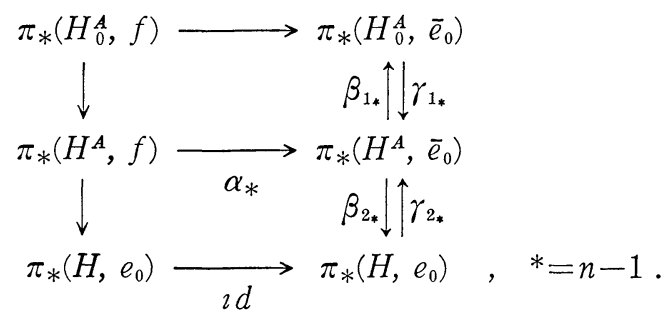

i. e. we have 
PROPOSITION 4. $\pi_{n-1}\left(H^{A}, f\right) \cong \pi_{n-1}\left(H, e_{0}\right) \times\left[S^{n-1} \wedge A, H\right]_{0}$ where $[,]_{0}$ denotes the set of homotopy classes in the base-point preserving category.

Now define an operation

$$
o:[A, H]_{0} \longrightarrow[G \wedge A, H]_{0}
$$

by the formula, for any map $s: A \rightarrow H$ with $s\left(a_{0}\right)=e_{o}$,

$$
o(s)(g \wedge a)=s(g a) s(a)^{-1} s\left(g a_{o}\right)^{-1} .
$$

Remark. If $A=G o(s)$ is the obstruction for the map $s$ being homomorphic up to homotopy.

LEMMA 5. Our obstruction $\hat{\xi}^{*}(f)\left(\in \pi_{n-1}\left(H^{A}, f\right)\right)$ is decomposed in Proposition 4 as follows:

$$
\alpha_{*}\left(\hat{\xi}^{*}(f)\right)=f_{*}\left(\lambda_{\xi}\right)+o(f)_{*}\left(\xi \wedge 1_{A}\right)
$$

where $\lambda_{\xi}=\partial_{\iota}$ for the boundary operator $\partial: \pi_{n}\left(S^{n}\right) \rightarrow \pi_{n-1}(A)$ in the homotopy exact sequence of the bundle.

Proof. The first factor easily follows from definitions, and the second follows from identities:

$$
\begin{aligned}
& o(f)_{*}\left(\xi \wedge 1_{A}\right)(x, a)=f(\xi(x) a) f(a)^{-1} f\left(\xi(x) a_{0}\right)^{-1} \text { and } \\
& \begin{aligned}
\beta_{1 *}\left[\alpha_{*}\left(\hat{\xi}^{*}(f)\right)\right](x, a) & =\alpha_{*}\left(\hat{\xi}^{*}(f)\right)(x, a)\left(\alpha_{*}\left(\hat{\xi}^{*}(f)\right)\left(x, a_{o}\right)\right)^{-1} \\
& =\hat{\xi}^{*}(f)(x, a) f(a)^{-1}\left[\hat{\xi}^{*}(f)\left(x, a_{o}\right) f\left(a_{o}\right)^{-1}\right]^{-1} \\
& =f(\xi(x) a) f(a)^{-1} f\left(a_{o}\right)^{+1} f\left(\xi(x) a_{o}\right)^{-1}
\end{aligned}
\end{aligned}
$$

As an application we have

Proposition 6. Let $p: X \rightarrow S^{n}$ be a principal G-bundle with its characteristic class $\xi$. Then if $f:\left(G, e_{0}\right) \rightarrow\left(H, e_{0}\right)$ is homomorphic up to homotopy $f$ is extendable over $X$ if and only if $f_{*}(\xi)=0$.

Proof. Since the operation " $o$ " is trivial for homomorphisms up to homotopy the proof is clear from Lemma 5.

\section{$\S 4$. The case of $Y$ being a $H$-space.}

Let $Y$ be a right $H$-space, i.e. the group $H$ acts on $Y$ from the right, and let $i: H \rightarrow Y$ be the map $i(h)=y_{0} h$. Now we consider a problem:

What is obstructions for extending a map $f:\left(A, a_{o}\right) \rightarrow\left(H, e_{0}\right)$ to a map $X \rightarrow Y$ ? Since our obstruction in $\S 1$ is natural for any map $Y \rightarrow Y^{\prime}$ one of obstruc- 
tions is given as the image of $\xi$ under the composite of homomorphisms:

where $f^{\prime}=i f$.

$$
\pi_{n-1}\left(G, e_{0}\right) \underset{\phi^{*}}{\longrightarrow} \pi_{n-1}\left(A^{A}, 1_{A}\right) \underset{f^{*}}{\longrightarrow} \pi_{n-1}\left(H^{A}, f\right) \underset{i^{*}}{\longrightarrow} \pi_{n-1}\left(Y^{A}, f^{\prime}\right)
$$

Thus we must describe the group $\pi_{n-1}\left(Y^{A}, f^{\prime}\right)$ with more usual concepts, and then we consider the following diagram which is analogus to the diagram in $\S 3$.

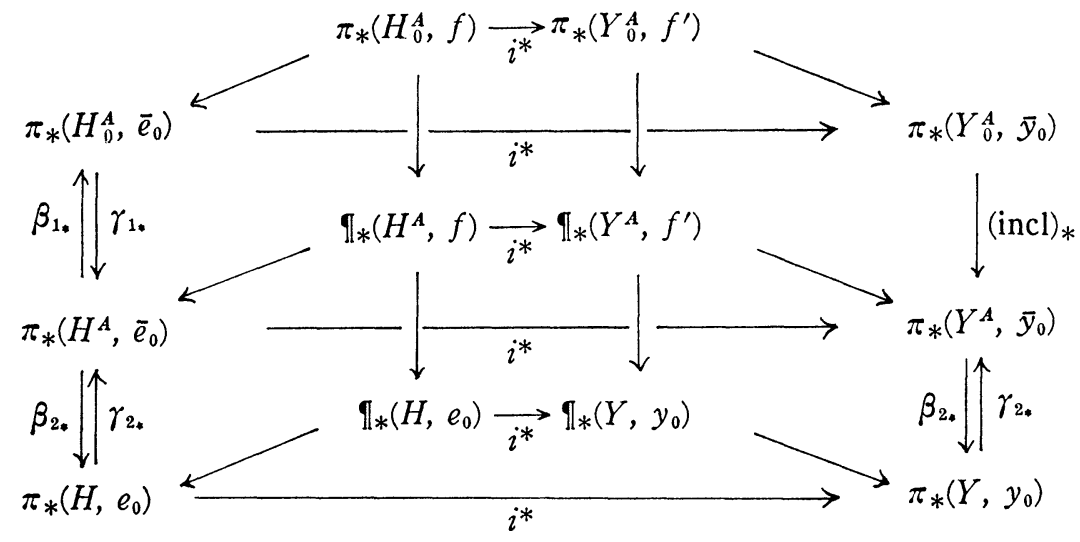

where $\pi_{*}$ denotes $\pi_{n-1}$.

Since the commutativity of the diagram is naturally obtained from the definitions we have

LeEma 7. A map $f:\left(A, a_{o}\right) \rightarrow\left(H, e_{0}\right)$ is extendable to a map $X \rightarrow Y$ if and only if

$$
\begin{aligned}
& f_{*}^{\prime}\left(\lambda_{\xi}\right)=0 \text { in } \pi_{n-1}\left(Y, y_{0}\right) \quad \text { and } \\
& i_{*}^{*}\left(o(f)_{*}\left(\xi \wedge 1_{A}\right)\right)=0 \quad \text { in }\left[S^{n-1} \wedge A, Y\right]_{0} .
\end{aligned}
$$

Proof.

$$
\begin{aligned}
i^{*}\left(\hat{\xi}^{*}(f)\right) & =i^{*} \alpha_{*}\left(\hat{\xi}^{*}(f)\right)=i^{*}\left(\gamma_{2_{*}}\left(f_{*}\left(\lambda_{\xi}\right)\right)\right)+\gamma_{1_{*}}\left(o(f)_{*}\left(\xi \wedge 1_{A}\right)\right) \\
& =f_{*}^{\prime}\left(\lambda_{\xi}\right)+(\text { incl. })_{*} i_{*}^{*}\left(o(f)_{*}\left(\xi \wedge 1_{A}\right)\right) .
\end{aligned}
$$

Thus $\beta_{2} \gamma_{2}=i d$. shows the proof.

Now, restating Lemma 7, we have

Proposition 8. A map $f:\left(A, a_{o}\right) \rightarrow\left(H, e_{0}\right)$ is extendable to a map $X \rightarrow Y$ if and only if $f_{*}\left(\lambda_{\xi}\right) \in \partial \pi_{n}(Y, H)$ and

$$
o(f)_{*}\left(\xi \wedge 1_{A}\right) \in \partial\left[D^{n} \wedge A, S^{n-1} \wedge A ; Y, H\right]_{o}
$$

As an application we have 
THEOREM 8. Let $p_{i}: X_{i} \rightarrow S^{n}$ be principal bundles having it's characteristic classes $\xi_{i}(i=1,2)$ respectively. Then a map $f:\left(G_{1}, e_{0}\right) \rightarrow\left(G_{2}, e_{0}\right)$ is extendable to a map $X_{1} \rightarrow X_{2}$ if and only if $f_{*}\left(\xi_{1}\right)=m \xi_{2}$ for some integer $m$ and $o(f)_{*}\left(\xi_{1} \wedge 1_{G_{1}}\right) \in$ $\partial\left[S^{n} \wedge G_{1}, S^{n}\right]_{0} \subset\left[S^{n-1} \wedge G_{1}, G_{2}\right]_{0}$.

And moreover as a special case we have

COROLlaRY 9. In Theorem 8 if $\operatorname{dim} G_{2}<n-1$ we can replace the second condition with $o(f)_{*}\left(\xi_{1} \wedge 1_{G_{1}}\right) \in \xi_{2^{*}}\left(\left[S^{n-1} \wedge G_{1}, S^{n-1}\right]_{0}\right)$.

COROLlaRy 10. In Corollary 9 we assume that $G_{1}, G_{2}$ are both path-connected. Then $X_{1}$ has the same homotopy type as $X_{2}$ if and only if there exists a homotopy equivalence $f: G_{1} \rightarrow G_{2}$ such that $f_{*}\left(\xi_{1}\right)= \pm \xi_{2}$ and $o(f)_{*}\left(\xi_{1} \wedge 1_{G_{1}}\right) \in \xi_{2^{*}}\left[S^{n-1} \wedge G_{1}, S^{n-1}\right]_{0}$.

Proofs now are clear from preceeding lemmas and propositions.

\section{§5. Examples.}

Example 1. Let $p: X_{\xi} \rightarrow S^{n}$ be a principal $S^{3}$-bundle with it's characteristic class $\xi\left(\in \pi_{n-1}\left(S^{3}\right), n \geqq 5\right)$ and let $f_{m}: S^{3} \rightarrow S^{3}$ be a map of degree $m$. Then $f_{m}$ is extendable to a map $X_{\xi} \rightarrow X_{\xi}$ if and only if $m(m-1) / 2\left(\tau E^{3} \xi\right) \in \xi \pi_{n+2}\left(S^{n-1}\right)$ where $\tau$ is Blaker-Massay map.

Proof. From Corollary 10 it is sufficient to compute $o\left(f_{m}\right)$. Let $c: S^{3} \wedge S^{3}$ $\rightarrow S^{3}$ be the commutator map, i.e. $c(x \wedge y)=x y x^{-1} y^{-1}$. Then from the definition of $o\left(f_{m}\right)$ (see $\S 3$ ) we have

$$
\begin{aligned}
o\left(f_{m}\right)(x \wedge y) & =f_{m}(x y) f_{m}(y)^{-1} f_{m}(x)^{-1} \\
& =(x y)^{m} y^{-m} x^{-m}=(x y)^{m-1}(x y) y^{-m} x^{-m}=(x y)^{m-1} x y^{-m+1} x^{-m} \\
& =(x y)^{m-1} y^{-m+1} x^{-m+1} x^{m-1} y^{m-1} x y^{1-m} x^{-m} \\
& =o\left(f_{m-1}\right)(x \wedge y) x^{m-1} y^{m-1} y^{1-m} x c\left(x^{-1} \wedge y^{m-1}\right) x^{-m} \\
& =o\left(f_{m-1}\right)(x \wedge y) x^{m} c\left(x^{-1} \wedge y^{m-1}\right) x^{-m} \\
& =o\left(f_{m-1}\right)(x \wedge y) c\left(x^{m} \wedge c\left(x^{-1} \wedge y^{m-1}\right)\right) c\left(x^{-1} \wedge y^{m-1}\right) .
\end{aligned}
$$

On the other hand we have

$$
c\left(x^{-1} \wedge y^{m-1}\right)=(1-m)_{\tau}
$$

for, $S^{6}=S^{3} \wedge S^{3} \underset{d}{\longrightarrow} S^{3} \wedge S^{3}=S^{6} \underset{c}{\longrightarrow} S^{3}$ is just the left side where $d$ is defined by $d(x \wedge y)=x^{-1} \wedge y^{m-1}$. Since the degree of $d$ is $1-m$ and the homotopy class of $c$ is Blaker-Massay class the proof is completed.

And moreover we have

$$
c\left(x^{m} \wedge c\left(x^{-1} \wedge y^{m-1}\right)\right)=0
$$


for, this map is factored as follows:

$$
S^{6}=S^{3} \wedge S^{3} \underset{t}{\longrightarrow} S^{3} \wedge S^{3} \wedge S^{3}=S^{9} \underset{1 \wedge c}{\longrightarrow} S^{3} \wedge S^{3} \underset{c}{\longrightarrow} S^{3}
$$

where $t$ is given by $t(x \wedge y)=x^{m} \wedge x^{-1} \wedge y^{m-1}$ :

Thus we have

$$
o\left(f_{m}\right)=o\left(f_{m-1}\right)+(1-m) \tau
$$

This shows the proof.

Example 2. For a principal $S^{3}$-bundle $p: X_{\xi} \rightarrow S^{n}(n \geqq 5)$, there exists an orientation reveresing homotopy equivalence $X_{\xi} \rightarrow X_{\xi}$ if and only if

$$
2 \xi=0 \text { and } \tau E^{3} \xi \in \xi \pi_{n+2}\left(S^{n-1}\right) .
$$

Example 3. For a principal $G$-bundle $p: X \rightarrow S^{n}$ we denote by $(X, G)^{(X, G)}$ the subspace of $X^{X},\left\{f \in X^{X} \mid f(G) \subset G\right\}$. Then there exists the fibring

$$
(X, G)^{(X, G)} \longrightarrow G^{G}
$$

which is obtained from the restriction of the domain of maps.

This gives rise the exact sequence of the sets with distinguished elements:

$$
\begin{aligned}
\pi_{o}\left((X, G)^{(X, G)}, 1_{G}\right) & \stackrel{\tau}{\longrightarrow} \pi_{o}\left(G^{G}, 1_{G}\right) \\
& \stackrel{\lambda}{\longrightarrow} \pi_{n-1}(G) /\{\xi\}+\left[S^{n-1} \wedge G, G\right] / \xi\left[S^{n-1} \wedge G, S^{n-1}\right]
\end{aligned}
$$

where $\lambda([f])=\left[f_{*}(\xi)\right]+\left[o(f)_{*}\left(\xi \wedge 1_{G}\right)\right]$.

\section{REFERENCES}

[1] I. M. James And J.H.C. Whitehead, The homotopy theory of sphere bundles over spheres, I, Proc. Lond. Math. Soc., 1954, 196-218.

[2] N.E. Steenrod, Topology of Fibre Bundles., Princeton Univ. Press, 1951.

Dept. of Math.

TOKYO INST. OF TECH.

MEgURO-KU TOKYO JAPAN

and Math. Inst. Univ. Heidelberg 